\title{
Association between Stress and Temporomandibular Disorder
}

\author{
Harukazu Kanehira, DDS, PhD, Akinori Agariguchi, DDS, PhD, Hisashi Kato, DDS, PhD, \\ Shigeki Yoshimine, DDS, and Hiroshi Inoue, DDS, PhD \\ Department of Removable Prosthodontics and Occlusion, Osaka Dental University, Osaka, Japan
}

\section{Clinical significance}

Temporomandibular disorder, a functional disorder of the temporomandibular joint, is an important area of investigation in prosthodontics because it connects directly with masticatory dysfunction. This study shows that psychological factors such as stress have an influence on temporomandibular disorder.

\begin{abstract}
Purpose: To evaluate the causes of temporomandibular disorder (TMD) by examining the relationships between 3 major TMD symptoms, parafunction, and stress, a questionnaire survey was performed during dental examinations in corporations.

Methods: The survey was performed using 6 questions on the following topics: 1 . Trismus; 2. Joint noise; 3. Pain; 4. Clenching in the daytime; 5 . Nocturnal bruxism; and 6. Stress.

There were 3,225 subjects, 2,809 males and 416 females and the mean age of the subjects was 40.12 years. The relationships between questions 1 to 5 and question 6 were examined by the chi-square test.

Results: There were significant correlations (question $1, p=0.001$; questions $2-5, p<0.001$ ).

Conclusion: Clearly, there was an influence of psychological factors, such as stress, on TMD, and such factors were considered to play important roles in its etiology, progression, and treatment. The results of this study suggest that well-controlled studies of TMD are necessary.
\end{abstract}

Key words: temporomandibular disorder (TMD), stress, etiology

\section{Introduction}

Occlusion, abnormalities in masticatory muscles,

\author{
Corresponding to: Dr Harukazu Kanehira \\ Department of Removable Prosthodontics and Occlusion, \\ Osaka Dental University \\ 1-5-17 Otemae, Chuo-ku, Osaka 540-0008, Japan \\ Tel: +81-6-6910-1517, Fax: +81-6-6910-1044 \\ E-mail: kanehira@cc.osaka-dent.ac.jp
}

Received on April 20, 2007/Accepted on December 14, 2007 and organic changes, such as internal derangement, have been considered to induce temporomandibular disorder (TMD) causing dysfunction of jaw joints. ${ }^{1-7}$ Furthermore, involvement of parafunction, such as bruxism, has been reported. ${ }^{7-13}$ The mechanism of TMD has been examined based on the classification of pain. ${ }^{14}$ With changes in the etiology of TMD, involvement of occlusal factors alone in TMD has been re-examined, ${ }^{15-19}$ while psychosocial factors have been evaluated. ${ }^{8,12,20-27}$

Laskin (1969) reported that the major cause of TMD is muscular hyperactivity induced by psychological stress and persistently poor oral habits, and that muscular fatigue causes spasm, resulting in temporomandibular displacement. ${ }^{28}$ Laskin further noted that this displacement causes occlusal abnormality, articular deformation, and muscular alteration, leading to organic disorder. ${ }^{28}$ The evaluation of TMD based on psychological factors has been considered important. ${ }^{14}$ However, there have been few reports of evaluation of TMD based on psychological factors in population-based studies. ${ }^{14,26}$

In the present study, we performed a questionnaire survey to evaluate the causes of TMD by examining the relationships between 3 major TMD symptoms, parafunction and stress.

\section{Materials and Methods}

An interview survey was performed using a questionnaire, consisting of age, sex, and the following questions:

Question 1: Do you sometimes have difficulties in opening your mouth?

Question 2: Are you aware of noise during opening and closing your mouth?

Question 3: Do you experience pain in or around the jaw joints during opening and closing your mouth?

Question 4: Do you clench your teeth in the day- 
Table 1 Results of questions 1-6.

\begin{tabular}{lrrrrrc}
\hline & Q1 & \multicolumn{1}{c}{ Q2 } & \multicolumn{1}{c}{ Q3 } & \multicolumn{1}{c}{ Q4 } & \multicolumn{1}{c}{ Q5 } & Q6 \\
\hline Yes & 191 & 712 & 114 & 387 & 679 & 1,473 \\
No & 3,034 & 2,513 & 3,111 & 2,838 & 2,546 & 1,752 \\
\hline
\end{tabular}

Table 2 Relationships between the results of questions 1-5 and question 6 .

\begin{tabular}{|c|c|c|c|c|c|c|c|c|c|c|c|}
\hline & & \multicolumn{2}{|c|}{ Q1 } & \multicolumn{2}{|c|}{ Q2 } & \multicolumn{2}{|c|}{ Q3 } & \multicolumn{2}{|c|}{ Q4 } & \multicolumn{2}{|c|}{ Q5 } \\
\hline & & Yes & No & Yes & No & Yes & No & Yes & No & Yes & No \\
\hline \multirow{2}{*}{ Q6 } & Yes & 109 & 1,364 & 382 & 1,091 & 72 & 1,401 & 247 & 1,226 & 403 & 1,070 \\
\hline & No & 82 & 1,670 & 330 & 1,422 & 42 & 1,710 & 140 & 1,612 & 276 & 1,476 \\
\hline
\end{tabular}

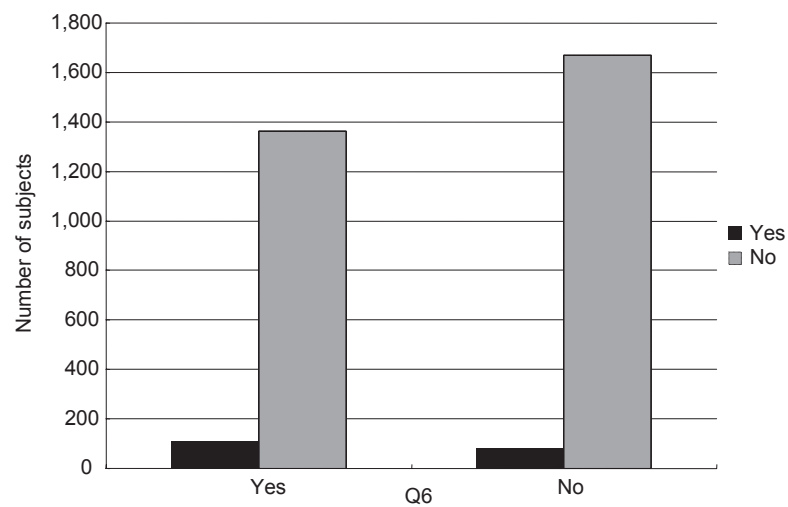

Fig. 1 Relationship between stress (question 6) and trismus (question 1).

Number of subjects, $\square$ Yes, $\square$ No.

time or at work?

Question 5: Do you clench or grind your teeth while asleep?

Question 6: Do you generally feel stress?

The subjects answered these questions with yes or no. With regard to questions 4 and 5, the answers were regarded as no when the subjects were unaware of clenching and bruxism. The subjects were asked to give answers about conditions at that time, independently of past signs and symptoms. All answers were subjective self-returns of subjects. The survey was performed between July 2005 and June 2006, and the total number of subjects was 3,225 . There were 2,809 males and 416 females and the mean age of the group was 40.12 years (range 19-64). The relationships between questions 1 to 5 and question 6 were examined by the chi-square test. The level of significance was set at $5 \%$. Analyses concerning sex and age groups were not performed in this study. This study protocol was approved by the Committee on Experimental Research on Humans of Osaka Dental University (No. 060715).

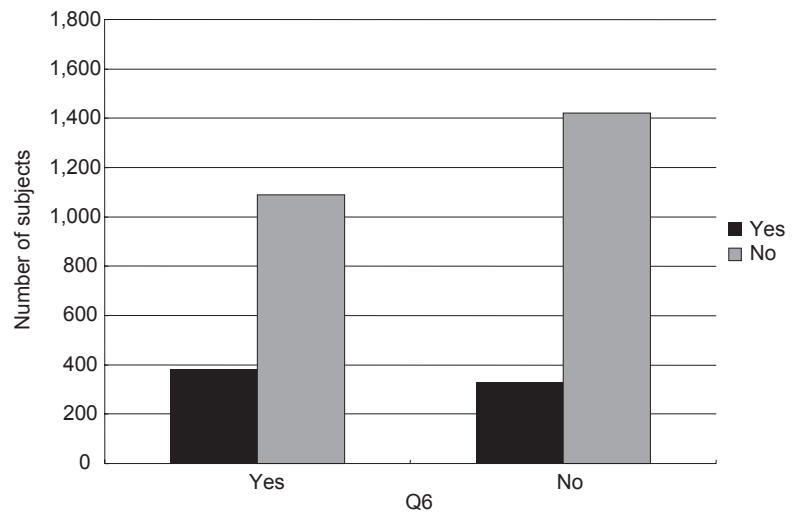

Fig. 2 Relationship between stress (question 6) and joint noise (question 2).

\section{Results}

\section{Data tabulation}

Table 1 shows the results. Of the 3,225 subjects, $191(5.9 \%)$ answered yes to question $1,712(22.1 \%)$ to question $2,114(3.5 \%)$ to question $3,387(12 \%)$ to question $4,679(21.1 \%)$ to question 5 , and 1,473 (45.7\%) to question 6.

\section{Analysis by the chi-square test}

Questions 1 and 6

One hundred and ninety-one subjects answered yes to question 1 (Do you sometimes have difficulties in opening your mouth?). Of the 191 subjects, 109/1,473 (7.4\%) answered yes to question 6 (Do you generally feel stress?). This number was significantly larger than $4.7 \%(82 / 1,752)$ who answered no $\left(p=0.001, \chi^{2}=10.6\right.$; Fig. 1, Tables 1 and 2$)$.

Questions 2 and 6

Of the 712 subjects who answered yes to question 2 (Are you aware of noise during opening and closing your mouth?), 25.9\% (382/1,473) answered question 6 with yes, and 18.8\% $(330 / 1,752)$ with no. The difference was significant $\left(p<0.001, \chi^{2}=\right.$ 23.4; Fig. 2, Tables 1 and 2). 


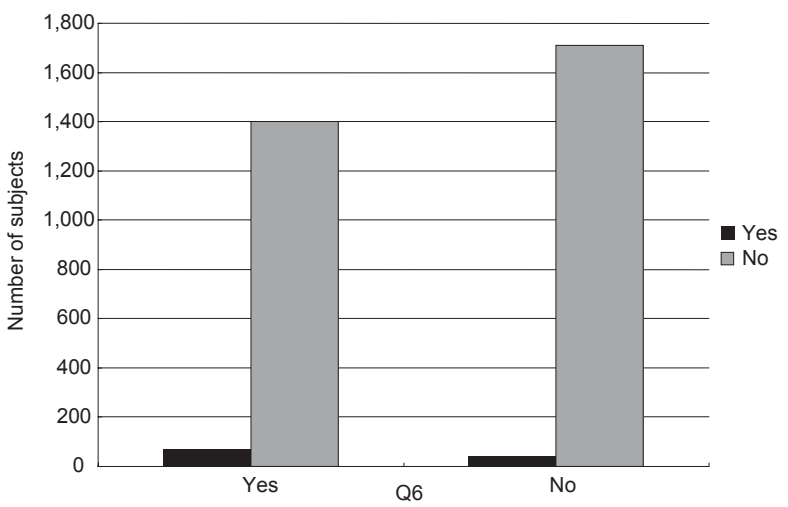

Fig. 3 Relationship between stress (question 6) and pain (question 3).

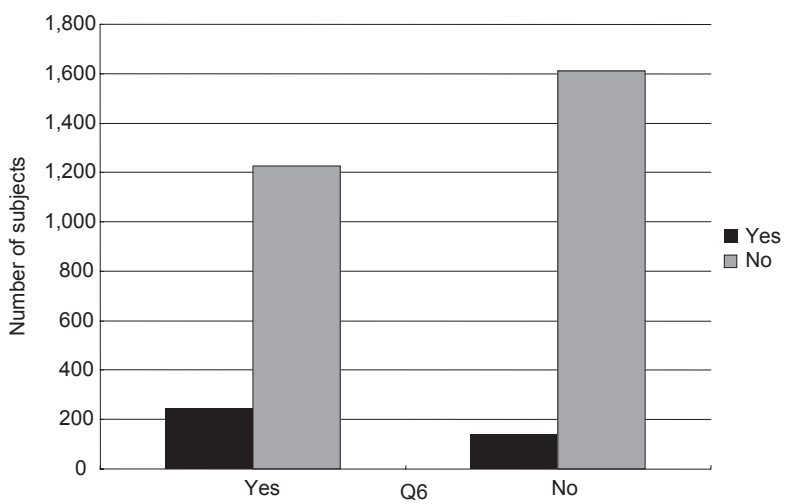

Fig. 4 Relationship between stress (question 6) and clenching (question 4).

\section{Questions 3 and 6}

With regard to question 3 (Do you experience pain in or around the jaw joints during opening and closing your mouth?), 114 subjects answered yes, and 3,111 no. Of the 114 subjects, 4.9\% $(72 / 1,473)$ answered question 6 with yes, and the remaining $2.4 \%(42 / 1752)$ with no. The difference was significant $\left(p<0.001, \chi^{2}=14.6\right.$; Fig. 3 , Tables 1 and 2).

Questions 4 and 6

With regard to question 4 (Do you clench your teeth in the daytime or at work?), 387 subjects answered yes, and 2,838 no. Of the 387 subjects, 247/1,473 (16.8\%) and 140/1752 (8.0\%) answered question 6 with yes and no, respectively, with a significant difference $\left(p<0.001, \chi^{2}=58.4\right.$; Fig. 4 , Tables 1 and 2).

Questions 5 and 6

Six hundred and seventy-nine subjects answered question 5 with yes, and 2,546 with no. Of the 679 subjects, 403/1,473 (27.4\%) answered question 6 with yes, and $276(15.8 \%)$ with no. The difference

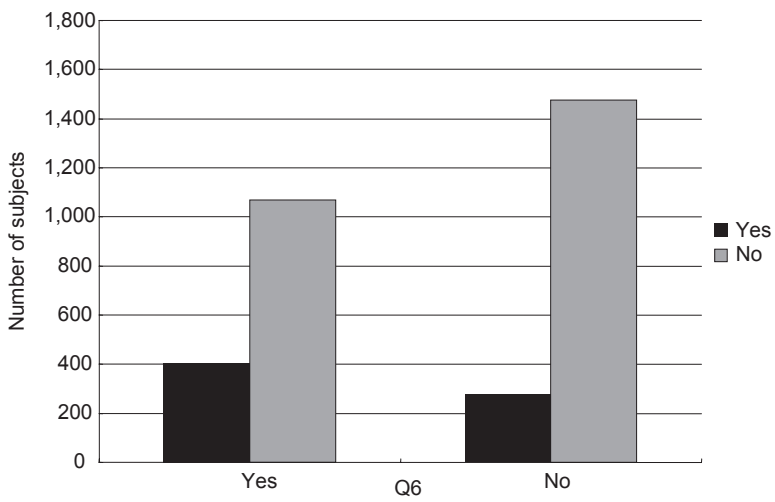

Fig. 5 Relationship between stress (question 6) and bruxism (question 5).

was significant $\left(p<0.001, \chi^{2}=64.8\right.$; Fig. 5 , Tables 1 and 2).

\section{Discussion}

\section{Data tabulation}

There have been a number of studies related to questions 1 to 3 , which correspond to 3 major TMD symptoms. ${ }^{12,16,17,26,29-37}$ However, since data reported by such studies ranged widely, it was difficult to compare them with the results of our study. In other words, since the investigation methods, number of subjects, and their age in this study differed from those of past studies, comparisons of data alone are insignificant. ${ }^{36}$ Comparisons of question 6 as a psychological factor ${ }^{8,12,14,18,20-28,38-45}$ and questions 4 and 5 as parafunctional factors, ${ }^{8-13,21,25,38,40,44}$ which are considered to be related to TMD, are also insignificant. The present survey was performed on dental examinations in corporations, and the investigation time was limited. Therefore, the subjects were asked to answer simple questions. Such a survey will always be affected by investigation methods or populations. . $^{10,14,22,39}$ To minimize such effects, international standards, such as the TMJ scale, should be used. , $24,29,34^{2}$ Furthermore, the investigation method has to be easily understood within a short time. ${ }^{11,12}$ The importance of population-based studies rather than adult patients-based studies has been reported. ${ }^{14,26,46}$ In the present study, the subjects were 3,225 general healthy adults, who worked at corporations, indicating that this survey was appropriate.

\section{Analysis by the chi-square test}

Analysis of the data by the chi-square test demon- 
strated that questions 1 to 5 were significantly correlated with question 6 (question $1, p=0.001$, Fig. 1 ; question $2, p<0.001$, Fig. 2 ; question $3, p<0.001$, Fig. 3; question $4, p<0.001$, Fig. 4 ; question $5, p<$ 0.001, Fig. 5, Tables 1 and 2), indicating that stress affected the factors shown by questions 1 to 5 .

Over the past 20 years, the involvement of occlusal factors in TMD as its etiology has been reexamined. ${ }^{14-19}$ This is because of the difficulty in study design unification and reproducibility. How ${ }^{-}$ ever, there have also been studies showing multiple and partial involvement of occlusal factors in TMD. ${ }^{7,9,12,14,15,35}$

There seems little doubt that parafunctional factors are involved in TMD, but it has not been clarified whether these factors alone or their combination with other factors is involved. ${ }^{8-11,21,25,38,40,41}$ In particular, it is necessary to perform a survey with strict methods for the detection of bruxism and clenching as parafunctional factors. ${ }^{13,39}$

In the 1990s, studies showing that psychological factors, such as stress, are involved in TMD increased. ${ }^{14}$ It has been reported that increases in stress induce a somatic reaction, leading to a linear increase in TMD. ${ }^{21,23,41}$ It has also been reported that stress conditions vary depending on whether TMD is present, and hyperfunction develops into parafunction, i.e., bruxism and clenching. ${ }^{38,39}$ Stud- $^{-}$ ies performed using the TMJ scale, by which detailed evaluation of psychological factors, such as stress, is considered possible, indicated that TMD was significantly correlated with such factors. ${ }^{24,29}$ Some studies suggested that these factors were involved in TMD based on the evaluation of hormones and somnipathy, but in many studies on the involvement of psychological factors, such as stress, the relationship between TMD and pain was examined. . $^{3,20,24,26,42}$ Since the pain level varies with sex, age, and occupation, it is always individually biased, suggesting that this parameter is pathologically inappropriate. . $2,27,42,47$ Furthermore, since stress is strongly affected by an individual's coping ability, its level could be changed by other factors, such as occlusal factors, indicating the necessity of consideration of their interactions and other risk factors. ${ }^{8,25,48,49}$

In epidemiological studies, like ours, it is necessary to analyze populations in detail. ${ }^{10,12,13,22,36,39}$ However, with regard to TMD, evaluation of its expression in general populations is considered more important than evaluation of this disorder. Various complicated TMD signs and symptoms should be etiologically analyzed in a large population by community diagnosis and health. ${ }^{26}$ To perform such analyses, carefully designed, analytical, cross-sectional, and population-based studies are required..$^{14}$

There is a "chicken-and egg" problem in trying to decide whether these psychological states have caused the functional problems, or are caused by them, or if any relationship exists at all. Psychological factors play an important role in the etiology, progression, and treatment of TMD. ${ }^{8}$ In the future, psychology-based therapies for TMD will be important and necessary.

\section{Conclusion}

Stress was significantly correlated with the $3 \mathrm{ma}^{-}$ jor TMD symptoms and parafunction. In other words, psychological factors, such as stress, play important roles in TMD, suggesting that such factors and well-controlled studies should be taken into consideration in TMD treatment.

\section{References}

1. Forssel H, Kirveskari P, Kangasniemi P. Effect of occlusal adjustment on mandibular dysfunction : A double-blind study. Acta Odontol Scand 1986; 44: 63-69.

2. Agerberg G, Bergenholtz A. Craniomandibular disorders in adult populations of West Bothnia, Sweden. Acta Odontol Scand 1989; 47: 129-140.

3. Abubaker AO, Raslan WF, Sotereanos GC. Estrogen and progesterone receptors in temporomandibular joint disc of symptomatic and asymptomatic persons: A preliminary study. J Oral Maxillofacial Surg 1993; 51: 1096-1100.

4. Ash MM. Philosophy of occlusion: Past and present. Dent Clin North Am 1995; 39: 233-255.

5. Okerson JP. Occlusion and functional disorder of the masticatory system. Dent Clin North Am 1995; 39: 285-300.

6. Cooper BC. The role of bioelectronic instruments in documenting and managing temporomandibular disorders. J Am Dent Assoc 1996; 127: 1611-1614.

7. Allen EP, Bayne SC, Becker IM et al. Annual review of selected dental literature: Report of the Committee on Scientific Investigation of the American Academy of Restorative Dentistry. J Prosthet Dent 2000; 84: 59-92.

8. Greene CS, Olson RE, Laskin DM. Psychological factors in the etiology, progression, and treatment of MPD syndrome. J Am Dent Assoc 1982; 105: 443-448. 
9. Nielsen IL, Ogro J, McNeill C et al. Alteration in proprioceptive reflex control in subjects with craniomandibular disorders. J Craniomandib Disord Facial Oral Pain 1987; 1: 170-178.

10. Moss RA, Lombardo TW, Villarosa GA et al. Oral habits and TMJ dysfunction in facial pain and nonpain subjects. J Oral Rehabil 1995; 22: 79-81.

11. Liu ZJ, Kubota S, Yamagata K et al. Quantitative and multidimensional evaluation of symptoms and correlative factors for temporomandibular disorders in clinical and subclinical subjects. J Jpn Orthod Soc 1996; 55: 445-460.

12. Conti PCR, Ferreira PM, Pegorano LF et al. A cross-sectional study of prevalence and etiology of signs and symptoms of temporomandibular disorders in high school and university students. J Orofacial Pain 1996; 10: 254-262.

13. Glaros AG, Tabacchi KN, Glass EG. Effect of parafunctional clenching on TMD pain. J Orofacial Pain 1998; 12: 145-152.

14. National Institutes of Health technology assessment conference statement. Management of temporomandibular disorders. J Am Dent Assoc 1996; 127: 1595-1606.

15. Droukas B, Lindee C, Carlsson GE. Occlusion and mandibular dysfunction: A clinical study of patients referred for functional disturbances of the masticatory system J Prosthet Dent 1985; 53: 402-406.

16. Eriksson E, Carlsson GE, Magnusson T. A long-term epidemiologic study of the relationship between occlusal factors and mandibular dysfunction in children and adolescents J Dent Res 1987; 66: 67-71.

17. Pullinger AG, Seligman DA, Solberg WK. Temporomandibular disorders. Part II: Occlusal factors associated with temporomandibular joint tenderness and dysfunction J Prosthet Dent 1988; 59: 363-367.

18. Lipp MJ. Temporomandibular symptoms and occlusion: A review of the literature \& the concept. NY State J 1990; 56: 58-66.

19. Pullinger AG, Seligman DA, Gornbein JA. Multiple logistic regression analysis of the risk and relative odds of temporomandibular disorders as a function of common occlusal features. J Prosthet Dent 1993; 72: 968-979.

20. Geissler PR. An investigation of the stress factor in the mandibular dysfunction syndrome. J Dent 1985; 13: 283-287.

21. Pingitore G, Chrobak V, Petrie J. The social and psychologic factors of bruxism. J Prosthet Dent 1991; 65: 443-446.

22. Stockstill JW, Callahan CD. Personality hardness, anxiety, and depression as constructs of interest in the study of temporomandibular disorders J Craniomandib Disord Facial Oral Pain 1991; 5: 129-134.

23. Parker MW, Holmes EK, Terezhalmy GT. Personality characteristics of patients with temporomandibular disorders: Diagnostic and therapeutic implica- tions J Orofacial Pain 1993; 7: 337-344.

24. Levitt SR, McKinney MW. Validating the TMJ Scale in a national sample of 10,000 patients: Demographic and epidemiologic characteristics. J Orofacial Pain 1994; 8: 25-35.

25. Pierce CJ, Chrisman K, Bennett ME et al. Stress, anticipatory stress, and psychologic measures related to sleep bruxism. J Orofacial Pain 1995; 9: 51-56.

26. LeResche L. Epidemiology of temporomandibular disorders: Implication for the investigation of etiology factors. Crit Rev Oral Biol Med 1997; 8: 291-305.

27. Eli I. Gender differences in pain. J Orofacial Pain 2000; 14: 184-186.

28. Laskin DM. Etiology of the pain-dysfunction syndrome. J Am Dent Assoc 1969; 79: 147-153.

29. Levitt SR, Mckinney MW, Lundeen TF. The TMJ Scale: cross-validation and reliability studies. J Craniomandib Pract 1988; 6: 18-25.

30. Wänman A, Agerberg G. Temporomandibular joint sounds in adolescents: A longitudinal study. Oral Sueg Oral Med Oral Pathol 1990; 69: 2-9.

31. Dworkin SF, Huggins KH, LeResche L et al. Epidemiology of signs and symptoms in temporomandibular disorders: clinical signs in cases and controls. $\mathrm{J}$ Am Dent Assoc 1990; 120: 273-281.

32. Stockstill JW, Mohl ND. Evaluation of temporomandibular joint sounds. Diagnostic analysis and clinical implications. Dent Clin North Am 1991; 35: $75-88$.

33. Lipton JA, Ship JA, Larach-Robinson D. Estimated prevalence and distribution of reported orofacial pain in the United States. J Am Dent Assoc 1993; 124: 115-121.

34. De Kanter RJAM, Truin GJ, Burgersdijk RCW et al. Prevalence in the Dutch adult population and a metaanalysis of signs and symptoms of temporomandibular disorder. J Dent Res 1993; 72: 1509-1518.

35. McNamara JA, Seligman DA, Okeson JP. Occlusion, orthodontic treatment, and temporomandibular disorders: A review. J Orofacial Pain 1995; 9: 73-90.

36. Carlsson GE. Epidemiology and treatment need for temporomandibular disorders. J Orofacial Pain 1999; 13: 232-237.

37. Arima T, Svensson P. Experimental grinding in healthy subjects: A model for postexercise jaw muscle soreness? J Orofacial Pain 1999; 13: 104-114.

38. Parker MW. A dynamic model of etiology in temporomandibular disorders. J Am Dent Assoc 1990; 120: 283-290.

39. Rugh JD, Montgomery GT. Physiological reaction of patients with TM disorders vs symptom-free controls on a physical stress task. J Craniomandib Disord Facial Oral Pain 1987; 1: 243-250.

40. Hung GJ, LeResche L, Critchlow CW et al. Risk factors for diagnostic subgroups of painful temporomandibular disorder. J Dent Res 2002; 81: 284-288.

41. Beaton RD, Egan KJ, Nakagawa-Kogan H et al. 
Self-reported symptoms of stress with temporomandibular disorder: Comparisons to healthy men and women. J Prosthet Dent 1991; 65: 289-293.

42. Dao TTT, LeResche L. Gender differences in pain. J Orofacial Pain 2000; 14: 169-184.

43. Bailey DR. Sleep disorders. Dent Clin North Am 1997; 41: 189-209.

44. Rosales VP, Ikeda K, Hizaki K et al. Emotional stress and brux-like activity of the masseter muscle in rats. Eur J Orthod 2002; 24: 107-117.

45. Southwell J, Deary IJ, Geissler P. Personality and anxiety in temporomandibular joint syndrome $\mathrm{pa}^{-}$ tients. J Oral Rehabil 1990; 17: 239-243.

46. Giamberardino MA. Gender differences in pain. J Orofacial Pain 2000; 14: 187-190.

47. Unruh AM. Gender variations in clinical pain experience. Pain 1996; 65: 123-167.

48. Schiffman EL, Fricton JR, Haley D. The relationship of occlusion, parafunctional habits and recent life events to mandibular dysfunction in a non-patient population. J Oral Rehabil 1992; 19: 201-223.

49. Wexler GB, Steed PA. Psychological factors and temporomandibular outcomes. J Craniomandib Pract 1998; 16: 72-77. 